Supporting Information

\title{
Cyclooxygenase-2 Inhibitors. 1,5-Diarylpyrrol-3-acetic Esters with Enhanced Inhibitory Activity Toward Cyclooxygenase-2 and Improved Cyclooxygenase-2/ Cyclooxygenase-1 Selectivity
}

Mariangela Biava, Giulio Cesare Porretta, Giovanna Poce, Sibilla Supino, Stefano Forli, Michele Rovini, Andrea Cappelli, Fabrizio Manetti, Maurizio Botta, Lidia Sautebin, Antonietta Rossi, Carlo Pergola, Carla Ghelardini, Elisa Vivoli, Francesco Makovec, Paola Anzellotti, Paola Patrignani, Maurizio Anzini

\section{Table of Contents:}

- Physicochemical, spectroscopical, and analytical data of compounds 9-16 


\section{Chemistry}

1-[4-(Fluoro)phenyl]pentane-1,4-dione (14a). Yellowish needles (yield 75\%). Analytical data, mp, and ${ }^{1} \mathrm{H}$ NMR spectrum were consistent with those reported in the literature. ${ }^{1}$

1-(Phenyl)pentane-1,4-dione (14d). Yellowish needles (yield 78\%). Analytical data, mp, and ${ }^{1} \mathrm{H}$ NMR spectrum were consistent with those reported in the literature. ${ }^{2}$

1-[4-(Methyl)phenyl]pentane-1,4-dione (14e). Yellowish needles (yield 76\%). Mp $60{ }^{\circ} \mathrm{C},{ }^{1} \mathrm{H}$ NMR $\left(\mathrm{CDCl}_{3}\right) 7.86(\mathrm{~d}, 2 \mathrm{H}, J=7.27 \mathrm{~Hz}), 7.26(\mathrm{~d}, 2 \mathrm{H}, J=7.27 \mathrm{~Hz}), 3.23$ (t, J=6.7 Hz, 2H), 2.87 (t, J=6.7 Hz, $2 \mathrm{H}), 2.42(\mathrm{~s}, 3 \mathrm{H}), 2.25$ (s, 3H). Anal. $\left(\mathrm{C}_{12} \mathrm{H}_{14} \mathrm{O}_{2}\right) \mathrm{C}, \mathrm{H}, \mathrm{O}$.

1-[4-(Trifluoromethyl)phenyl]pentane-1,4-dione (14f). Yellowish needles (yield 75\%). Analytical data, mp, and ${ }^{1} \mathrm{H}$ NMR spectrum were consistent with those reported in the literature. ${ }^{3}$

2-Methyl-5-[4-(methylsulfonyl)phenyl]-1-[4-(fluoro)phenyl]-1H-pyrrole (11a). White needles (yield $82 \%$ ). Analytical data, mp, and ${ }^{1} \mathrm{H}$ NMR spectrum were consistent with those reported in the literature. $^{1}$

2-Methyl-5-[4-(methylsulfonyl)phenyl]-1-[3,4-(difluoro)phenyl]-1H-pyrrole (11b). White needles (yield 74\%). Analytical data, mp, and ${ }^{1} \mathrm{H}$ NMR spectrum were consistent with those reported in the literature. $^{1}$

2-Methyl-5-[4-(methylsulfonyl)phenyl]-1-[3-(fluoro)phenyl]-1H-pyrrole (11d). White needles (yield 80\%). ${ }^{1} \mathrm{H}$ NMR $\left(\mathrm{CDCl}_{3}\right)$ : 7.65-7.68 (d, 2H), 7.36-7.37 (m, 1H), 7.23-7.25 (d, 2H), 6.88-6.95 (m, $3 \mathrm{H}), 6.51-6.57(\mathrm{~s}, 1 \mathrm{H}), 4.79-4.82(\mathrm{~s}, 1 \mathrm{H}), 2.16(\mathrm{~s}, 3 \mathrm{H})$. Anal. $\left(\mathrm{C}_{18} \mathrm{H}_{15} \mathrm{FNO}_{2} \mathrm{~S}\right) \mathrm{C}, \mathrm{H}, \mathrm{N}, \mathrm{S}, \mathrm{O}, \mathrm{F}$.

2-Methyl-1-[4-(methylsulfonyl)phenyl]-5-[4-(fluoro)phenyl]-1H-pyrrole (15a). White needles (yield $86 \%$ ). Analytical data, mp, and ${ }^{1} \mathrm{H}$ NMR spectrum were consistent with those reported in the literature. $^{1}$

2-Methyl-1-[4-(methylsulfonyl)phenyl]-5-[3,4-(difluoro)phenyl]-1H-pyrrole (15b). Yellowish needles (yield 78\%). Mp $179{ }^{\circ} \mathrm{C},{ }^{1} \mathrm{H}$ NMR $\left(\mathrm{CDCl}_{3}\right)$ 7.98(m, 2H), $7.26(\mathrm{~m}, 2 \mathrm{H}), 6.96(\mathrm{~m}, 1 \mathrm{H}), 6.92(\mathrm{~m}$, 1H), $6.68(\mathrm{~m}, 1 \mathrm{H}), 6.32(\mathrm{~d}, 1 \mathrm{H}, J=3.5 \mathrm{~Hz}), 6.11(\mathrm{~d}, 1 \mathrm{H}, J=3.5 \mathrm{~Hz}), 3.11(\mathrm{~s}, 3 \mathrm{H}), 2.16$ (s, 3H). Anal. $\left(\mathrm{C}_{18} \mathrm{H}_{15} \mathrm{NO}_{2} \mathrm{SF}_{2}\right) \mathrm{C}, \mathrm{H}, \mathrm{N}, \mathrm{O}, \mathrm{F}, \mathrm{S}$.

2-Methyl-1-[4-(methylsulfonyl)phenyl]-5-(phenyl)-1H-pyrrole (15d). Yellowish needles (yield 83\%). Mp $144{ }^{\circ} \mathrm{C},{ }^{1} \mathrm{H}$ NMR $\left(\mathrm{CDCl}_{3}\right)$ 7.93(d, 2H, $\left.J=7.27 \mathrm{~Hz}\right), 7.25$ (d, 2H, J=7.27 Hz), $7.14(\mathrm{~m}, 3 \mathrm{H})$, $7.00(\mathrm{~m}, 2 \mathrm{H}), 6.36(\mathrm{~d}, 1 \mathrm{H}, J=3.5 \mathrm{~Hz}), 6.13(\mathrm{~d}, 1 \mathrm{H}, J=3.5 \mathrm{~Hz}), 3.10(\mathrm{~s}, 3 \mathrm{H}), 2.18$ (s, 3H). Anal. $\left(\mathrm{C}_{18} \mathrm{H}_{17} \mathrm{NO}_{2} \mathrm{~S}\right) \mathrm{C}, \mathrm{H}, \mathrm{N}, \mathrm{O}, \mathrm{S}$. 
2-Methyl-1-[4-(methylsulfonyl)phenyl]-5-[4-(methyl)phenyl]-1H-pyrrole (15e). Yellowish needles (yield 80\%). Mp $168{ }^{\circ} \mathrm{C},{ }^{1} \mathrm{H}$ NMR $\left(\mathrm{CDCl}_{3}\right)$ 7.68(d, 2H, J=7.27 Hz), 7.22 (d, 2H, J=7.27 Hz), 7.09 (d, $2 \mathrm{H}, J=7.27 \mathrm{~Hz}), 6.93(\mathrm{~d}, 2 \mathrm{H}, J=7.27 \mathrm{~Hz}), 6.50(\mathrm{~d}, 1 \mathrm{H}, J=3.5 \mathrm{~Hz}), 6.12(\mathrm{~d}, 1 \mathrm{H}, J=3.5 \mathrm{~Hz}), 3.86(\mathrm{~s}, 3 \mathrm{H})$, $3.02(\mathrm{~s}, 3 \mathrm{H}), 2.12$ (s, 3H). Anal. $\left(\mathrm{C}_{19} \mathrm{H}_{19} \mathrm{NO}_{2} \mathrm{~S}\right) \mathrm{C}, \mathrm{H}, \mathrm{N}, \mathrm{O}, \mathrm{S}$.

2-Methyl-1-[4-(methylsulfonyl)phenyl]-5-[4-(trifluoro)phenyl]-1H-pyrrole (15f). Yellowish needles (yield 78\%). Mp $170{ }^{\circ} \mathrm{C},{ }^{1} \mathrm{H}$ NMR $\left(\mathrm{CDCl}_{3}\right) 7.90$ (m, 2H), $7.22(\mathrm{~m}, 2 \mathrm{H}), 7.10$ (m, 2H), 6.90 (m, $2 \mathrm{H}), 6.50$ (d, 1H, J=3.5 Hz), 6.12 (d, 1H, J=3.5 Hz), 3.10 (s, 3H), 2.10 (s, 3H). Anal. $\left(\mathrm{C}_{19} \mathrm{H}_{16} \mathrm{NO}_{2} \mathrm{SF}_{3}\right)$ C, H, N, S, O, F.

Ethyl-2-methyl-5-[4-(methylsulfonyl)phenyl]-1-[3,4-(difluoro)phenyl]-1H-pyrrol-3-glyoxylate (12b). Yellowish needles (yield 77\%). Mp $140{ }^{\circ} \mathrm{C},{ }^{1} \mathrm{H}$ NMR $\left(\mathrm{CDCl}_{3}\right) 7.77(\mathrm{~m}, 2 \mathrm{H}), 7.25$ (m, 3H), 7.05 (m, 2H), 6.95 (s, 1H), 4.41 (q, 2H, J= $7.1 \mathrm{~Hz}), 3.01$ (s, 3H), 2.46 (s, 3H), 1.42 (t, 3H, J=7.1 Hz). Anal. $\left(\mathrm{C}_{22} \mathrm{H}_{19} \mathrm{NO}_{5} \mathrm{SF}_{2}\right) \mathrm{C}, \mathrm{H}, \mathrm{N}, \mathrm{F}, \mathrm{O}, \mathrm{S}$.

Ethyl-2-methyl-5-[4-(methylsulfonyl)phenyl]-1-[4-(methoxy)phenyl]-1H-pyrrol-3-glyoxylate (12c). Yellowish needles (yield 75\%). Mp $177{ }^{\circ} \mathrm{C},{ }^{1} \mathrm{H}$ NMR $\left(\mathrm{CDCl}_{3}\right) 7.66$ (d, 2H, J=7.27 Hz), 7.19 (d, 2H, J=7.27 Hz), 7.07 (d, 2H, J=7.27 Hz), 7.04 (d, 2H, J=7.27 Hz), 6.53 (s, 1H), 4.19 (q, 2H, J= 7.1 Hz), $3.0(\mathrm{~s}, 3 \mathrm{H}), 2.42(\mathrm{~s}, 3 \mathrm{H}) 2.07(\mathrm{~s}, 3 \mathrm{H}), 1.25$ (t, 3H, J=7.1 Hz) Anal. $\left(\mathrm{C}_{23} \mathrm{H}_{23} \mathrm{NO}_{6} \mathrm{~S}\right) \mathrm{C}, \mathrm{H}, \mathrm{N}, \mathrm{O}, \mathrm{S}$.

Ethyl-2-methyl-5-[4-(methylsulfonyl)phenyl]-1-[3-(fluoro)phenyl]-1H-pyrrol-3-glyoxylate (12d). Yellowish needles (yield 57\%) Mp $140{ }^{\circ} \mathrm{C},{ }^{1} \mathrm{H}$ NMR $\left(\mathrm{CDCl}_{3}\right)$ : 7.66-7.72 (d, 2H), 7.37-7.38 (m, 1H), 7.23-7.25 (d, 2H), 6.90-6.93 (m, 3H), $6.00(\mathrm{~s}, 1 \mathrm{H}), 4.20$ (q, 2H, J = 7.1 Hz), 2.86 (s, 3H), 2.16 (s, 3H), $1.30(\mathrm{t}, 3 \mathrm{H}, J=7.1 \mathrm{~Hz})$. Anal. $\left(\mathrm{C}_{22} \mathrm{H}_{20} \mathrm{NO}_{5} \mathrm{SF}\right) \mathrm{C}, \mathrm{H}, \mathrm{N}, \mathrm{S}, \mathrm{O}, \mathrm{F}$.

Ehyl-2-methyl-1-[4-(methylsulfonyl)phenyl]-5-[3,4-(difluoro)phenyl]-1H-pyrrol-3-glyoxylate (16b). Yellowish needles (yield 80\%). Mp $136{ }^{\circ} \mathrm{C},{ }^{1} \mathrm{H}$ NMR $\left(\mathrm{CDCl}_{3}\right) 8.03$ (m, 2H), 7.33 (m, 2H), 6.93 $(\mathrm{m}, 2 \mathrm{H}), 6.71(\mathrm{~m}, 1 \mathrm{H}), 6.70(\mathrm{~s}, 1 \mathrm{H}), 4.40(\mathrm{q}, 2 \mathrm{H}, J=7.1 \mathrm{~Hz}), 3.10(\mathrm{~s}, 3 \mathrm{H}), 2.45(\mathrm{~s}, 3 \mathrm{H}), 1.42(\mathrm{t}, 3 \mathrm{H}$, $J=7.1 \mathrm{~Hz})$. Anal. $\left(\mathrm{C}_{22} \mathrm{H}_{19} \mathrm{NO}_{5} \mathrm{SF}_{2}\right) \mathrm{C}, \mathrm{H}, \mathrm{N}, \mathrm{S}, \mathrm{O}, \mathrm{F}$.

Ethyl-2-methyl-1-[4-(methylsulfonyl)phenyl]-5-[4-(methoxy)phenyl]-1H-pyrrol-3-glyoxylate (16c). Yellowish needles (yield 70\%). Mp $132{ }^{\circ} \mathrm{C},{ }^{1} \mathrm{H}$ NMR $\left(\mathrm{CDCl}_{3}\right) 7.95$ (d, 2H, J=7.27 Hz), 7.33 (d, 2H, J=7.27 Hz), $7.00(\mathrm{~d}, 2 \mathrm{H}, J=7.27 \mathrm{~Hz}), 6.95(\mathrm{~d}, 2 \mathrm{H}, J=7.27 \mathrm{~Hz}), 6.88(\mathrm{~s}, 1 \mathrm{H}), 4.41$ (q, 2H, J=7.1 $\mathrm{Hz}), 3.75$ (s, 3H), 3.12 (s, 3H), 2.47 (s, 3H), 1.42 (t, 3H, J=7.1 Hz). Anal. $\left(\mathrm{C}_{23} \mathrm{H}_{23} \mathrm{NO}_{6} \mathrm{~S}\right) \mathrm{C}, \mathrm{H}, \mathrm{N}, \mathrm{O}, \mathrm{S}$. Ethyl-2-methyl-1-[4-(methylsulfonyl)phenyl]-5-(phenyl)-1H-pyrrol-3-glyoxylate (16d). Yellowish needles (yield 72\%). Mp $140{ }^{\circ} \mathrm{C},{ }^{1} \mathrm{H} \mathrm{NMR}\left(\mathrm{CDCl}_{3}\right) 8.00$ (d, 2H, J=7.27 Hz), 7.35 (d, 2H, J=7.27 Hz), 
$7.17(\mathrm{~m}, 3 \mathrm{H}), 6.99(\mathrm{~m}, 2 \mathrm{H}), 6.93(\mathrm{~s}, 1 \mathrm{H}), 4.40(\mathrm{q}, 2 \mathrm{H}, J=7.1 \mathrm{~Hz}), 3.09(\mathrm{~s}, 3 \mathrm{H}), 2.48(\mathrm{~s}, 3 \mathrm{H}), 1.42(\mathrm{t}$, $3 \mathrm{H}, J=7.1 \mathrm{~Hz})$. Anal. $\left(\mathrm{C}_{22} \mathrm{H}_{21} \mathrm{NO}_{5} \mathrm{~S}\right) \mathrm{C}, \mathrm{H}, \mathrm{N}, \mathrm{O}, \mathrm{S}$.

Ethyl-2-methyl-1-[4-(methylsulfonyl)phenyl]-5-[4-(methyl)phenyl]-1H-pyrrol-3-glyoxylate (16e). Yellowish needles (yield 74\%). Mp $182{ }^{\circ} \mathrm{C},{ }^{1} \mathrm{H}$ NMR $\left(\mathrm{CDCl}_{3}\right) 7.99$ (d, 2H, J=7.27 Hz), 7.32 (d, 2H, $J=7.27 \mathrm{~Hz}), 6.98(\mathrm{~d}, 2 \mathrm{H}, J=7.27 \mathrm{~Hz}), 6.87(\mathrm{~d}, 2 \mathrm{H}, J=7.27 \mathrm{~Hz}), 6.85(\mathrm{~s}, 1 \mathrm{H}), 4.42(\mathrm{q}, 2 \mathrm{H}, J=7.1 \mathrm{~Hz})$, $3.10(\mathrm{~s}, 3 \mathrm{H}), 2.48(\mathrm{~s}, 3 \mathrm{H}), 2.28(\mathrm{~s}, 3 \mathrm{H}), 1.40(\mathrm{t}, 3 \mathrm{H}, \mathrm{J}=7.1 \mathrm{~Hz})$. Anal. $\left(\mathrm{C}_{23} \mathrm{H}_{23} \mathrm{NO}_{5} \mathrm{~S}\right) \mathrm{C}, \mathrm{H}, \mathrm{N}, \mathrm{O}, \mathrm{S}$.

Ethyl-2-methyl-1-[4-(methylsulfonyl)phenyl]-5-[4-(trifluoro)phenyl]-1H-pyrrol-3-glyoxylate (16f). Yellowish needles (yield 70\%). Mp $145^{\circ} \mathrm{C},{ }^{1} \mathrm{H}$ NMR $\left(\mathrm{CDCl}_{3}\right) 7.92$ (m, 2H), 7.51 (m, 2H,), 7.48 (m, 2H), $7.41(\mathrm{~m}, 2 \mathrm{H}), 6.12(\mathrm{~s}, 1 \mathrm{H}), 4.17(\mathrm{q}, 2 \mathrm{H}, J=7.1 \mathrm{~Hz}), 2.84(\mathrm{~s}, 3 \mathrm{H}), 2.14(\mathrm{~s}, 3 \mathrm{H}), 1.33(\mathrm{t}, 3 \mathrm{H}$, $J=7.1 \mathrm{~Hz})$. Anal. $\left(\mathrm{C}_{23} \mathrm{H}_{20} \mathrm{NO}_{5} \mathrm{SF}_{3}\right) \mathrm{C}, \mathrm{H}, \mathrm{N}, \mathrm{S}, \mathrm{O}, \mathrm{F}$.

Ethyl-2-methyl-5-[4-(methylsulfonyl)phenyl]-1-[3,4-(difluoro)phenyl]-1H-pyrrol-3-acetate (1b). Yellowish needles (yield 60\%). Mp $156{ }^{\circ} \mathrm{C},{ }^{1} \mathrm{H}$ NMR $\left(\mathrm{CDCl}_{3}\right) 7.69$ (m, 2H), 7.22 (m, 3H), 7.15 (m, 1H), $7.02(\mathrm{~m}, 1 \mathrm{H}), 6.50(\mathrm{~s}, 1 \mathrm{H}), 4.19(\mathrm{q}, 2 \mathrm{H}, J=7.1 \mathrm{~Hz}), 3.49(\mathrm{~s}, 2 \mathrm{H}), 3.01(\mathrm{~s}, 3 \mathrm{H}), 2.07(\mathrm{~s}, 3 \mathrm{H}), 1.29(\mathrm{t}$, $3 \mathrm{H}, \mathrm{J}=7.1 \mathrm{~Hz})$. Anal. $\left(\mathrm{C}_{22} \mathrm{H}_{21} \mathrm{NO}_{4} \mathrm{SF}_{2}\right) \mathrm{C}, \mathrm{H}, \mathrm{N}, \mathrm{S}, \mathrm{O}, \mathrm{F}$.

Ethyl-2-methyl-5-[4-(methylsulfonyl)phenyl]-1-[4-(methoxy)phenyl]-1H-pyrrol-3-acetate (1c). Yellow oil, (yield 30\%). ${ }^{1} \mathrm{H}$ NMR $\left(\mathrm{CDCl}_{3}\right): 7.66$ (d, 2H, J=7.27 Hz), 7.19 (d, 2H, J=7.27 Hz), 7.07 (d, 2H, J=7.27 Hz), 7.04 (d, 2H, J=7.27 Hz), 6.53 (s, 1H), 4.19 (q, 2H, J = 7.1), 3.50 (s, 2H), 3.0 (s, 3H), 2.40 (s, 3H) 2.07 (s, 3H), 1.29 (t, 3H, J = 7.1Hz). Anal. $\left(\mathrm{C}_{23} \mathrm{H}_{25} \mathrm{NO}_{5} \mathrm{~S}\right) \mathrm{C}, \mathrm{H}, \mathrm{N}, \mathrm{O}, \mathrm{S}$.

Ethyl-2-methyl-5-[4-(methylsulfonyl)phenyl]-1-[3-(fluoro)phenyl]-1H-pyrrol-3-acetate (1d). Yellowish needles (yield 30\%). $\mathrm{Mp} 132{ }^{\circ} \mathrm{C},{ }^{1} \mathrm{H} \mathrm{NMR}\left(\mathrm{CDCl}_{3}\right)$ : 7.66-7.72 (d, 2H), 7.37-7.38 (m, 1H), 7.23-7.25 (d, 2H), 6.90-6.93 (m, 3H), $5.80(\mathrm{~s}, 1 \mathrm{H}), 4.12(\mathrm{q}, 2 \mathrm{H}, J=7.1 \mathrm{~Hz}), 3.39(\mathrm{~s}, 2 \mathrm{H}), 2.84(\mathrm{~s}, 3 \mathrm{H})$, $2.16(\mathrm{~s}, 3 \mathrm{H}), 1.30(\mathrm{t}, 3 \mathrm{H}, J=7.1 \mathrm{~Hz})$. Anal. $\left(\mathrm{C}_{22} \mathrm{H}_{22} \mathrm{NO}_{4} \mathrm{SF}\right) \mathrm{C}, \mathrm{H}, \mathrm{N}, \mathrm{S}, \mathrm{O}, \mathrm{F}$.

Ehyl-2-methyl-1-[4-(methylsulfonyl)phenyl]-5-[3,4-(difluoro)phenyl]-1H-pyrrol-3-acetate

(2b). Yellowish needles (yield 65\%). Mp $143{ }^{\circ} \mathrm{C},{ }^{1} \mathrm{H}$ NMR $\left(\mathrm{CDCl}_{3}\right) 7.96$ (m, 2H), 7.29 (m, 2H), 6.94 (m, 1H), $6.79(\mathrm{~m}, 1 \mathrm{H}), 6.66(\mathrm{~m}, 1 \mathrm{H}), 6.35(\mathrm{~s}, 1 \mathrm{H}) 4.18(\mathrm{q}, 2 \mathrm{H}, J=7.1 \mathrm{~Hz}), 3.48(\mathrm{~s}, 2 \mathrm{H}), 3.13(\mathrm{~s}, 3 \mathrm{H}), 2.09$ (s, $3 \mathrm{H}), 1.27$ (t, 3H, J=7.1 Hz). Anal. $\left(\mathrm{C}_{22} \mathrm{H}_{21} \mathrm{NO}_{4} \mathrm{SF}_{2}\right) \mathrm{C}, \mathrm{H}, \mathrm{N}, \mathrm{S}, \mathrm{O}, \mathrm{F}$.

Ethyl-2-methyl-1-[4-(methylsulfonyl)phenyl]-5-[4-(methoxy)phenyl]-1H-pyrrol-3-acetate (2c). Yellowish oil, (yield 65\%). ${ }^{1} \mathrm{H} \mathrm{NMR}\left(\mathrm{CDCl}_{3}\right) 7.95$ (d, 2H, J=7.27 Hz), 7.33 (d, 2H, J=7.27 Hz), 7.00 (d, 2H,J=7.27 Hz), 6.95 (d, 2H,J=7.27 Hz), 6.52 (s, 1H), 4.18 (q, 2H, J=7.1 Hz), 3.47 (s, 2H), 3.0 (s, $3 \mathrm{H}), 2.42(\mathrm{~s}, 3 \mathrm{H}) 2.08(\mathrm{~s}, 3 \mathrm{H}), 1.29(\mathrm{t}, 3 \mathrm{H}, J=7.1 \mathrm{~Hz})$ Anal. $\left(\mathrm{C}_{23} \mathrm{H}_{25} \mathrm{NO}_{5} \mathrm{~S}\right) \mathrm{C}, \mathrm{H}, \mathrm{N}, \mathrm{S}, \mathrm{O}$. 
Ethyl-2-methyl-1-[4-(methylsulfonyl)phenyl]-5-(phenyl)-1H-pyrrol-3-acetate (2d). Yellowish needles (yield 65\%). Mp $110{ }^{\circ} \mathrm{C},{ }^{1} \mathrm{H}$ NMR $\left(\mathrm{CDCl}_{3}\right) 7.93(\mathrm{~d}, 2 \mathrm{H}, J=7.27 \mathrm{~Hz}), 7.32$ (d, 2H, J=7.27 Hz), $7.13(\mathrm{~m}, 3 \mathrm{H}), 6.98(\mathrm{~m}, 2 \mathrm{H}), 6.37(\mathrm{~s}, 1 \mathrm{H}), 4.19(\mathrm{q}, 2 \mathrm{H}, J=7.1 \mathrm{~Hz}), 3.50(\mathrm{~s}, 2 \mathrm{H}), 3.08(\mathrm{~s}, 3 \mathrm{H}), 2.11(\mathrm{~s}$, $3 \mathrm{H}), 1.25$ (t, 3H, J = 7.1). Anal. $\left(\mathrm{C}_{22} \mathrm{H}_{23} \mathrm{NO}_{4} \mathrm{~S}\right) \mathrm{C}, \mathrm{H}, \mathrm{N}, \mathrm{S}, \mathrm{O}$.

Ethyl-2-methyl-1-[4-(methylsulfonyl)phenyl]-5-[4-(methyl)phenyl]-1H-pyrrol-3-acetate

(2e).

Yellowish needles (yield 50\%). Mp $108{ }^{\circ} \mathrm{C},{ }^{1} \mathrm{H}$ NMR $\left(\mathrm{CDCl}_{3}\right) 7.90$ (d, 2H, J=7.27 Hz), 7.30 (d, 2H, $J=7.27 \mathrm{~Hz}), 6.95(\mathrm{~d}, 2 \mathrm{H}, J=7.27 \mathrm{~Hz}), 6.88$ (d, 2H, J=7.27 Hz), 6.33 (s, 1H), 4.17 (q, 2H, J = 7.1), 3.49 $(\mathrm{s}, 2 \mathrm{H}), 3.00(\mathrm{~s}, 3 \mathrm{H}), 2.25(\mathrm{~s}, 3 \mathrm{H}), 2.11(\mathrm{~s}, 3 \mathrm{H}), 1.28(\mathrm{t}, 3 \mathrm{H}, \mathrm{J}=7.1 \mathrm{~Hz})$. Anal. $\left(\mathrm{C}_{23} \mathrm{H}_{25} \mathrm{NO}_{4} \mathrm{~S}\right) \mathrm{C}, \mathrm{H}, \mathrm{N}$, S, O.

Ethyl-2-Methyl-1-[4-(methylsulfonyl)phenyl]-5-[4-(trifluoro)phenyl]-1H-pyrrol-3-acetate (2f). Yellowish needles (yield 65\%). Mp $165{ }^{\circ} \mathrm{C},{ }^{1} \mathrm{H}$ NMR $\left(\mathrm{CDCl}_{3}\right) 7.90$ (m, 2H), 7.53 (m, 2H,), 7.45 (m, 2H), $7.42(\mathrm{~m}, 2 \mathrm{H}), 5.81(\mathrm{~s}, 1 \mathrm{H}), 4.12(\mathrm{q}, 2 \mathrm{H}, J=7.1 \mathrm{~Hz}), 3.39(\mathrm{~s}, 2 \mathrm{H}), 2.84(\mathrm{~s}, 3 \mathrm{H}), 2.14(\mathrm{~s}, 3 \mathrm{H}), 1.30(\mathrm{t}$, $3 \mathrm{H}, J=7.1 \mathrm{~Hz})$. Anal. $\left(\mathrm{C}_{23} \mathrm{H}_{22} \mathrm{NO}_{4} \mathrm{SF}_{3}\right) \mathrm{C}, \mathrm{H}, \mathrm{N}, \mathrm{S}, \mathrm{O}, \mathrm{F}$.

\section{References}

1. Khanna, I. K.; Weier, R. M.; Paul, Y. Y.; Collins, W.; Miyashiro, J. M.; Koboldt, C. M.; Veenhuizen, A. W.; Currie, J. L.; Seibert, K.; Isakson, P. C. 1,2-Diarylpyrroles as Potent and Selective Inhibitors of Cyclooxygenase-2. J. Med. Chem. 1997, 40, 1619-1633.

2. Xue, S.; Li, L.-Z.; Liu, Y.-K.; Guo, Q.-X. Zinc-Mediated Chain Extension Reaction of 1,3Diketones to 1,4-Diketones and Diastereoselective Synthesis of trans-1,2-Disubstituted Cyclopropanols. J. Org. Chem. 2006, 71, 215-218.

3. Avery, T. D.; Taylor, D. K.; Tiekink, E. R. T. A New Route to Diastereomerically Pure Cyclopropanes Utilizing Stabilized Phosphorus Ylides and $\gamma$-Hydroxy Enones Derived from 1,2Dioxines: Mechanistic Investigations and Scope of Reaction. J. Org. Chem. 2000, 65, 5531-5546. 


\begin{tabular}{|c|c|c|c|c|}
\hline \multirow{3}{*}{ Compd } & \multirow{3}{*}{ Formula } & $\mathbf{C}$ & $\mathbf{H}$ & $\mathbf{N}$ \\
\hline & & Calcd \% & Calcd \% & Calcd \% \\
\hline & & Found \% & Found \% & Found \% \\
\hline \multirow[t]{2}{*}{$\mathbf{1 a}$} & $\mathrm{C}_{22} \mathrm{H}_{22} \mathrm{FNO}_{4} \mathrm{~S}$ & 63.60 & 5.34 & 3.37 \\
\hline & & 63.55 & 5.28 & 3.30 \\
\hline \multirow[t]{2}{*}{$1 b$} & $\mathrm{C}_{22} \mathrm{H}_{21} \mathrm{~F}_{2} \mathrm{NO}_{4} \mathrm{~S}$ & 60.96 & 4.88 & 3.23 \\
\hline & & 60.89 & 4.86 & 3.28 \\
\hline \multirow[t]{2}{*}{ 1c } & $\mathrm{C}_{23} \mathrm{H}_{25} \mathrm{NO}_{5} \mathrm{~S}$ & 64.62 & 5.89 & 3.28 \\
\hline & & 64.55 & 5.90 & 3.41 \\
\hline \multirow[t]{2}{*}{ 1d } & $\mathrm{C}_{22} \mathrm{H}_{22} \mathrm{FNO}_{4} \mathrm{~S}$ & 63.60 & 5.34 & 3.37 \\
\hline & & 63.53 & 5.45 & 3.30 \\
\hline \multirow[t]{2}{*}{$2 \mathbf{a}$} & $\mathrm{C}_{22} \mathrm{H}_{22} \mathrm{FNO}_{4} \mathrm{~S}$ & 63.60 & 5.34 & 3.37 \\
\hline & & 63.65 & 5.31 & 3.43 \\
\hline \multirow[t]{2}{*}{$2 b$} & $\mathrm{C}_{22} \mathrm{H}_{21} \mathrm{~F}_{2} \mathrm{NO}_{4} \mathrm{~S}$ & 60.96 & 4.88 & 3.23 \\
\hline & & 60.88 & 4.82 & 3.17 \\
\hline \multirow[t]{2}{*}{$2 c$} & $\mathrm{C}_{23} \mathrm{H}_{25} \mathrm{NO}_{5} \mathrm{~S}$ & 64.62 & 5.89 & 3.28 \\
\hline & & 64.77 & 5.84 & 3.23 \\
\hline \multirow[t]{2}{*}{$2 d$} & $\mathrm{C}_{22} \mathrm{H}_{23} \mathrm{NO}_{4} \mathrm{~S}$ & 66.48 & 5.83 & 3.52 \\
\hline & & 66.34 & 5.71 & 3.65 \\
\hline \multirow[t]{2}{*}{$2 e$} & $\mathrm{C}_{23} \mathrm{H}_{25} \mathrm{NO}_{4} \mathrm{~S}$ & 67.13 & 6.12 & 3.40 \\
\hline & & 67.23 & 6.16 & 3.28 \\
\hline \multirow[t]{2}{*}{$2 f$} & $\mathrm{C}_{23} \mathrm{H}_{22} \mathrm{~F}_{3} \mathrm{NO}_{4} \mathrm{~S}$ & 59.35 & 4.76 & 3.01 \\
\hline & & 59.51 & 4.77 & 2.95 \\
\hline \multirow[t]{2}{*}{$12 a$} & $\mathrm{C}_{22} \mathrm{H}_{20} \mathrm{FNO}_{5} \mathrm{~S}$ & 61.53 & 4.69 & 3.26 \\
\hline & & 61.46 & 4.73 & 3.19 \\
\hline \multirow[t]{2}{*}{$12 b$} & $\mathrm{C}_{22} \mathrm{H}_{19} \mathrm{~F}_{2} \mathrm{NO}_{5} \mathrm{~S}$ & 59.05 & 4.28 & 3.13 \\
\hline & & 59.00 & 4.20 & 3.27 \\
\hline \multirow[t]{2}{*}{$12 \mathrm{c}$} & $\mathrm{C}_{23} \mathrm{H}_{23} \mathrm{NO}_{6} \mathrm{~S}$ & 62.57 & 5.25 & 3.17 \\
\hline & & 62.51 & 5.33 & 3.01 \\
\hline
\end{tabular}




$\begin{array}{ccccc}\text { 12d } & \mathrm{C}_{22} \mathrm{H}_{20} \mathrm{FNO}_{5} \mathrm{~S} & 61.53 & 4.69 & 3.26 \\ & & 61.80 & 4.75 & 3.19 \\ \text { 16a } & \mathrm{C}_{22} \mathrm{H}_{20} \mathrm{FNO}_{5} \mathrm{~S} & 61.53 & 4.69 & 3.26 \\ & & 61.87 & 4.62 & 3.26 \\ \text { 16b } & \mathrm{C}_{22} \mathrm{H}_{19} \mathrm{~F}_{2} \mathrm{NO}_{5} \mathrm{~S} & 59.05 & 4.28 & 3.13 \\ & & 58.98 & 4.01 & 3.18 \\ \text { 16c } & \mathrm{C}_{23} \mathrm{H}_{23} \mathrm{NO}_{6} \mathrm{~S} & 62.57 & 5.25 & 3.17 \\ & & 62.90 & 5.31 & 3.20 \\ \text { 16d } & \mathrm{C}_{22} \mathrm{H}_{21} \mathrm{NO}_{5} \mathrm{~S} & 64.22 & 5.14 & 3.40 \\ & & 64.41 & 5.28 & 3.23 \\ \text { 16e } & \mathrm{C}_{23} \mathrm{H}_{23} \mathrm{NO}_{5} \mathrm{~S} & 64.92 & 5.45 & 3.29 \\ & & 65.19 & 5.57 & 3.17 \\ \text { 16f } & \mathrm{C}_{23} \mathrm{H}_{20} \mathrm{~F}_{3} \mathrm{NO}_{5} \mathrm{~S} & 57.61 & 4.20 & 2.92 \\ & & 57.80 & 4.34 & 2.78\end{array}$

